\title{
Giant Magnetoelectricity in Aluminium Substituted Y-Hexaferrites
}

\author{
P. NOVÁK ${ }^{a, *}$, V. CHLAN $^{b}$ AND K. KNÍŽEK ${ }^{a}$ \\ ${ }^{a}$ Institute of Physics ASCR, Cukrovarnická 10, 16253 Praha, Czech Republic \\ ${ }^{b}$ Charles University, Faculty of Mathematics and Physics \\ V Holešovičkách 2, 18000, Praha 8, Czech Republic
}

\begin{abstract}
The electron structure of $\mathrm{Al}$ containing Y-hexaferrite that exhibits the giant magnetoelectricity is calculated. Results show strong preference of $\mathrm{Al}$ for octahedral sites. Orbital moment of some of the iron ions is found to be unusually large.
\end{abstract}

PACS numbers: 75.85.+t, 71.20.Ps, 75.50.Gg

\section{Introduction}

In a recent paper Chun et al. reported a giant magnetoelectricity (ME) in the $\mathrm{Al}$ substituted Y-hexaferrite $\mathrm{Ba}_{0.5} \mathrm{Sr}_{1.5} \mathrm{Zn}_{2}\left(\mathrm{Fe}_{1-x} \mathrm{Al}_{x}\right)_{12} \mathrm{O}_{22}$ [1]. For an optimum $x=$ 0.08 the ME effect is by an order of magnitude stronger and it occurs at much lower external magnetic field compared to the parent compound, in which ME was found earlier by Kimura et al. [2]. The surprising enhancement of $\mathrm{ME}$ by the $\mathrm{Al}$ substitution was tentatively ascribed to a decrease of the magnetocrystalline anisotropy, which keeps the magnetization in the plane perpendicular to the hexagonal axis. The working hypothesis of Chun et al. is that practically all $\mathrm{Al}$ ions substitute Fe in an octahedral sublattice $18 \mathrm{~h}$ in which the Fe position is off-center. There are three other octahedral sublattices $3 \mathrm{a}, 3 \mathrm{~b}$ and $6 \mathrm{c}$ filled by $\mathrm{Fe}$ in the parent compound. Two tetrahedral sublattices $6 c_{1}$ and $6 c_{2}$ are occupied by Fe and $\mathrm{Zn}$. Similarly as in $\mathrm{GaFeO}_{3}$ [3] the off-centricity may lead to a large orbital moment from which presumably the anisotropy originates. The effect of $\mathrm{Al}$ may then be either direct (decrease of the number of Fe, possesing large orbital momentum), or indirect by diminishing the off-centricity.

In the present contribution we find out to which extent the above described scenario is supported by an $a b$ initio calculation of the electron structure. In particular the total energy of the hexaferrites containing $\mathrm{Al}$ in different sublattices are compared, the off-centricity of the $\mathrm{Fe}$ is evaluated and the orbital momentum of iron is calculated.

\section{Method}

The problem is complicated by the fact that there is a lot of disorder in the system in question: $\mathrm{Ba}$ and $\mathrm{Sr}$ enter the same sublattice and the two tetrahedral sublattices

\footnotetext{
* corresponding author; e-mail: novakp@fzu.cz
}

are partially occupied by Zn and partially by Fe. Similarly as in our paper [4] we circumvented this problem by considering $\left(\mathrm{Ba}_{1-y} \mathrm{Sr}_{y}\right)_{2} \mathrm{Zn}_{2}\left(\mathrm{Fe}_{1-x} \mathrm{Al}_{x}\right)_{12} \mathrm{O}_{22}$ system for three Sr concentrations $y=0,0.5,1$ and two different distributions of $(\mathrm{Fe}, \mathrm{Zn})$. This distribution is characterized by parameter $\gamma$, which is likely to lie between 0.6 and 0.9 [5]. To preserve as high symmetry as possible the calculations were done for $\gamma=0.5$ and 1 . The WIEN2k package [6] was used and in order to describe better the strong correlation of the $3 d$ electrons of Fe and $\mathrm{Zn}$ the GGA $+U$ method was employed. The computational details are more completely described in Ref. [4]. The substitution of aluminium was modelled by replacing in the unit cell one Fe by $\mathrm{Al}$. This corresponds to $\mathrm{Al}$ concentration $x=0.0833$, which is close to the optimal value 0.08 found by Chun et al. [1]. The total energy was then minimized by varying the positions of the atoms.

\section{Results and discussion}

For all six combinations of $y$ and $\gamma$ we found that the lowest total energy corresponds to $\mathrm{Al}$ in octahedral sublattice 3b. Very close, however, is the energy of substitution in the sublattice $18 \mathrm{~h}$ (Fig. 1). The energy cost of $\mathrm{Al}$ substitution in remaining four sublattices is higher by at least $0.4 \mathrm{eV}$. Because there are six times more $18 \mathrm{~h}$ than $3 \mathrm{~b}$ sites, the difference $\approx 0.05 \mathrm{eV}$ of the total energies may actually mean that at the temperature of crystal growth most of $\mathrm{Al}$ ions enter sublattice $18 \mathrm{~h}$ and the calculation thus supports the assumption made by Chun et al. [1].

The Fe ions on $18 \mathrm{~h}$ sites are off-centered, the displacement being along the hexagonal axis. We characterize the off-center shift by parameter $\omega$ :

$$
\omega=\sum_{j=1,6}\left[z\left(\mathrm{O}_{j}\right)-z(\mathrm{Fe})\right]
$$

where $z\left(\mathrm{O}_{j}\right)$ is the $z$ coordinate of the nearest oxygen neighbor of the Fe in question. $\omega$ was evaluated for all six combinations of $y$ and $\gamma$ of the parent compound as 


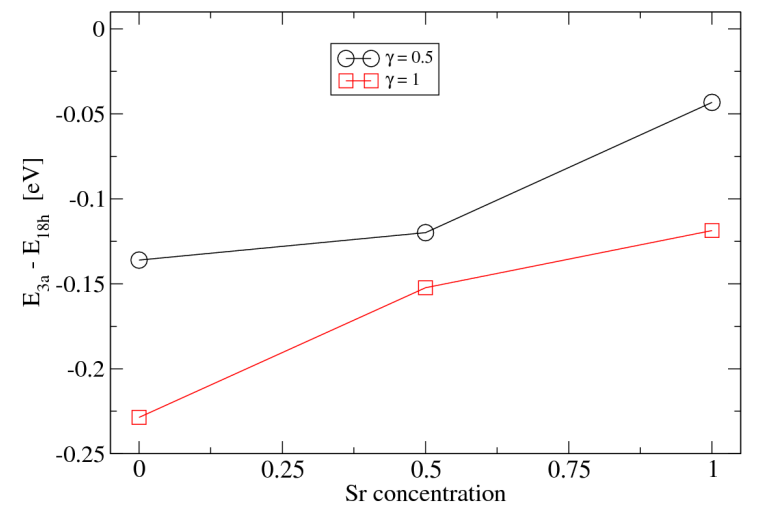

Fig. 1. The energy difference per formula unit of exchanging one $\mathrm{Fe}$ atom by $\mathrm{Al}$ in the sublattice $18 \mathrm{~h}$ and $3 \mathrm{~b}$.

well as for these six combinations with $\mathrm{Al}$ in $18 \mathrm{~h}$ sublattice. We found strong dependence of $\omega$ on $(\mathrm{Fe}, \mathrm{Zn})$ distribution (absolute value of $\omega$ for $\gamma=1 / 2$ was about $0.05 \mathrm{~nm}$, while for $\gamma=1$ it was approximately four times smaller). The dependence of $\omega$ on the Sr concentration is much weaker and also presence of $\mathrm{Al}$ has only small and unsystematic effect. The quenching of the off-center shift by presence of $\mathrm{Al}$ is therefore not supported by our calculation.

All above calculations were performed without the spin-orbit coupling (soc). In order to calculate the orbital moment the iteration procedure was first converged without soc, afterwards for several directions of the magnetization a single iteration was run that included soc and the orbital moment was determined. The magnitude of orbital moment $\boldsymbol{l}$ depends on both $\gamma$ and $x$, and, to a smaller extent, on the direction of the magnetization. In the unsubstituted compounds it may reach large value, considering that nominally all iron ions are trivalent with half-filled $3 d$ shell. The biggest $|\boldsymbol{l}|=0.23$ was found for $y=0.5, \gamma=0.5$ and $\mathrm{Fe}$ on the $6 \mathrm{c}$ sublattice, for Fe on $18 \mathrm{~h}$ sites $|\boldsymbol{l}|$ ranges between 0.05 and 0.14 . The substitution of $\mathrm{Al}$ leads to a significant reduction of $|\boldsymbol{l}|$ by $\approx 50 \%$ for
Fe on the $6 \mathrm{c}$ and $18 \mathrm{~h}$ sites. We did not analyze the reason for the reduction, but it is likely to be connected with the lower local symmetry in the $\mathrm{Al}$ substituted systems.

\section{Conclusions}

The results obtained corroborate two of the assumptions made by Chun et al.: large preference of $\mathrm{Al}$ for the octahedral sites and presence of iron ions with unusually large orbital moment, which is reduced by the $\mathrm{Al}$ substitution. To find out whether this leads to a smaller magnetocrystalline anisotropy is the subject of our ongoing investigation.

\section{Acknowledgments}

The work was supported by project IAA100100803 of the Grant Agency of the AS CR.

\section{References}

[1] S.H. Chun, Y.S. Chai, Y.S. Oh, D. Jaiswal-Nagar, S.Y. Haam, I. Kim, B. Lee, D.H. Nam, K.-T. Ko, J.-H. Park, J.-H. Chung, K.H. Kim, Phys. Rev. Lett. 104, 037204 (2010).

[2] T. Kimura, G. Lawes, A.P. Ramirez, Phys. Rev. Lett. 94, 137201 (2005).

[3] J.-Y. Kim, T.Y. Koo, J.-H. Park, Phys. Rev. Lett. 96, 047205 (2006).

[4] P. Novák, K. Knížek, J. Rusz, Phys. Rev. B 76, 024432 (2007).

[5] S. Utsumi, D. Yoshiba, N. Momozawa, J. Phys. Soc. Jpn. 76, 034704 (2007).

[6] P. Blaha, K. Schwarz, G.K.H. Madsen, D. Kvasnicka, J. Luitz, WIEN2k, An Augmented Plane Wave+Local Orbitals Program for Calculating Crystal Properties, Karlheinz Schwarz, Techn. Universität Wien, Austria 2001.

[7] K. Koư̌il, V. Chlan, H. Štěpánková, P. Novák, K. Knížek, J. Hybler, T. Kimura, Y. Hiraoka, J. Buršík, J. Magn. Magn. Mater. 322, 1243 (2010). 